\title{
Comparisons of Static, Quasi-Static and Dynamic 3D Porous Media Scale Network Models for Two-Phase Immiscible Flow in Porous Media
}

\author{
X. H. Zhang ${ }^{1, *}$, Q. J. $\mathrm{Liu}^{2}$, X. B. $\mathrm{Lu}^{1}$ \\ ${ }^{1}$ Institute of Mechanics, Chinese Academy of Sciences, Beijing 100080, China \\ ${ }^{2}$ Research Institute of Petroleum Exploration and Development Petro China, Beijing 100083, China \\ Email: lqj@petrochina.com.cn
}

\begin{abstract}
A dynamic 3D pore-scale network model is formulated for investigating the effect of interfacial tension and oil-water viscosity during chemical flooding. The model takes into account both viscous and capillary forces in analyzing the impact of chemical properties on flow behavior or displacement configuration, while the static model with conventional invasion percolation algorithm incorporates the capillary pressure only. From comparisons of simulation results from these models, it indicates that the static pore scale network model can be used successfully when the capillary number is low. With the capillary increases due to the enhancement of water viscosity or decrease of interfacial tension, only the quasi-static and dynamic model can give insight into the displacement mechanisms.
\end{abstract}

Key words: 3D Pore scale network model, relative permeability, interfacial tension.

\section{INTRODUCTION}

A pore scale network model as a computer stochastic-simulating technology in reservoir physics is attractive when it is required to simulate actual laboratory experiments. Although static and quasistatic network models have been used to describe a wide range of phenomena (e.g. capillary pressure characteristics, relative permeability curves et al), the dynamic network model is of important significance in currently Enhanced Oil Recovery (EOR) processes which is characterized by low interfacial tension (IFT) and comparatively high capillary number.

Dahel and Celia[1], JI-YOUN ARNS[2], Blunt and King[3], Fatt[4], Koplick and Lasseter[5], and Morrow[6] carried out a lot of correlative study on different characteristics of network model. The present work compares the results of the 3D dynamic network models, the static models and the quasi-static models. The capillary pressure curves and the relative permeability curves are obtained for three kinds of models. From comparisons of simulation results from these models, it indicates that the static pore scale network models can be used successfully when the capillary number is low. With the capillary increases due to the enhancement of water viscosity or decrease of interfacial tension, only the quasi-static and dynamic models can give insight into the displacement mechanisms. Although the computational is complex, it is shown that only the 3D dynamic network model can give a description of the interface movement occurs in capillary ducts, which had pronounced significance for chemical flooding.

\section{THE 3D PORE SCALE NETWORK MODEL}

A 3D pore scale network model with two kinds of cubic lattices was constructed (Figure 1) to represent low and high connectivity correspondingly, as show in Figure 1. The radii of the pore throats and the nodes are randomly distributed according to the mercury injection data from real core samples (Figure 2). 

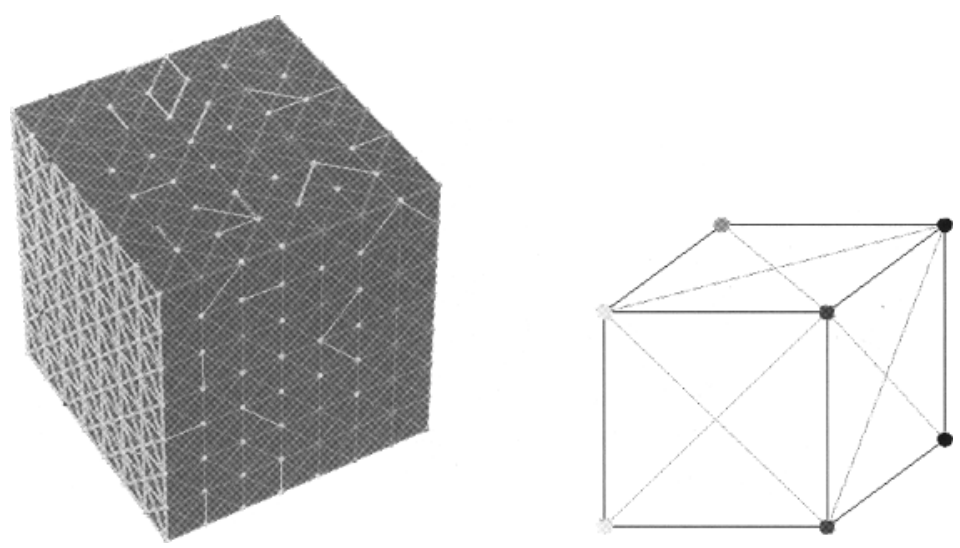

Figure 1: Model Construction

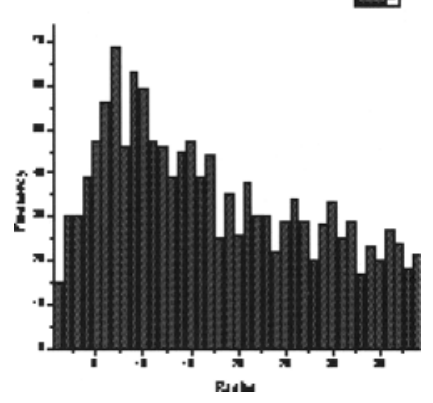

Figure 2: Pore throat radii distribution: truncated log-normal distribution

For a pore scale network model, both static and dynamic, the flow in the tube is governed by the Stokes' equation. For a single fluid, the volumetric flux is described by the following equation

$q_{i j}=-\frac{r_{i j}^{2}}{8 \mu_{i j} L_{i j}} \Delta p_{i j}$

In which $i$ and $j$ stand for pore $i$ and adjacent pore $j$, respectively. The volumetric flux at an interface is described as follows:

$q_{i j}=-\frac{r_{i j}^{2}}{8 \bar{\mu}_{i j} L_{i j}}\left(\Delta p_{i j}-P_{c}\right)$

where $P_{c}$ is the capillary pressure, $\bar{\mu}_{i j}$ is a variable of time.

Because the fluids are incompressible, the conservation of mass can be expressed in terms of integral of the volume flowing through the node $i$ in each time step

$\sum_{j \in N_{i}} Q_{i j}=0$

The static network model is based on invasion percolation: in drainage process the biggest throats are invaded first while in imbibing process the smallest throats are invaded first. Therefore, the capillary pressure governs the flow, while the viscous force is negligible. For the dynamic and quasi-static network models, the invasion percolation was governed by the competition of capillary force and viscous force. The interfaces are assumed to pass through the throats instantaneously in quasi-static 
model, while for dynamic model the position and movement of the interface was updated gradually with the percolation process.

One throat is allowed to be invaded once. The volumetric flux for a single fluid is the same as that of the dynamic models. However, when there will be an interface, the average viscous coefficient is adopted instead of the viscous coefficient changing with time.

\section{REULTS AND DISCUSSIONS}

Simulations are carried out with a network of size $8 \times 8 \times 8$. Initially the network is completely saturated with wetting fluid, another non-wetting phase were injected at inlet and the opposite side. as outlet.

Capillary number $\left(N_{c}\right)$ is defined as a dimensionless group representing the ratio of viscous to interfacial forces affecting the flow of fluid in porous media, as applied to water displacement of oil.

$N_{c}=\frac{\mu_{w} \nu_{w}}{\sigma \phi}$

For static model, percolation process was governed by capillary pressure only, so the effect of capillary number can not be simulated. The relative permeability curve is obtained (Figure 3).

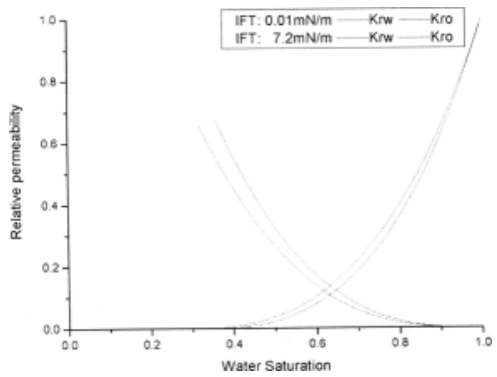

Figure 3: Relative permeability curve from static model

Quasi static and dynamic models relax the constraint that the capillary number be small. In theses two types model, the influence of viscous forces is modeled through an explicit computation of the pressure field in the network. For quasi static model, invasion percolation occurred instantaneously. Figure 4 gives the relations of the relative permeability of the quasi-static model with the IFT of 7.2 $\mathrm{mN} / \mathrm{m}$ and $1.0 \times 10^{-2} \mathrm{mN} / \mathrm{m}$. It is shown that the saturation at the intersection for the low IFT is greater than that of the high one and the height at the intersection is a little greater, which indicates that the relative permeability of the invasion phase will be greater when the IFT decreases.

Figure 5 gives the relations of the relative permeability of the dynamic model with the IFT of 7.2 $\mathrm{mN} / \mathrm{m}$ and $1.0 \times 10^{-2} \mathrm{mN} / \mathrm{m}$. It is shown that both the water and oil relative permeability changed greatly.

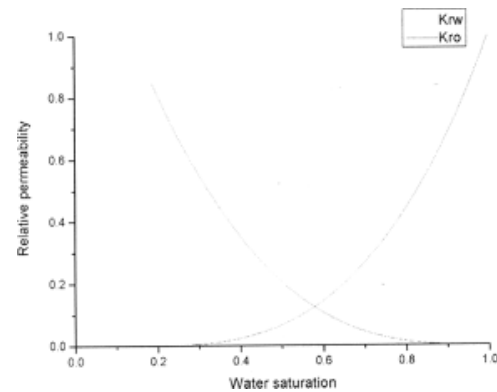

Figure 4: Relative permeability from quasi-static model 


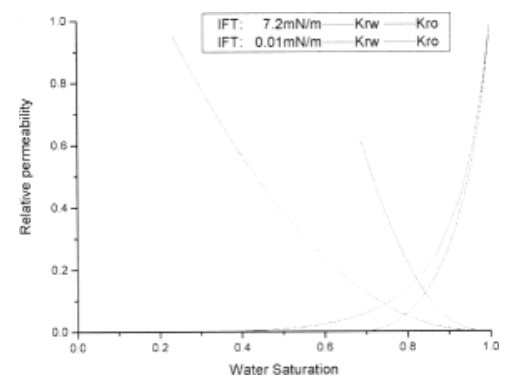

Figure 5: Relative permeability from dynamic model

It should be emphasized that this paper does not attempt to give some understanding of IFT effect on permeability or to validate the dynamic model with reproduced experimental results. Instead, this work only intends to give some comparisons among these modcls.

\section{CONCLUSION}

In the paper, it is shown that when dealing with low capillary numbers, static models, quasi-static models and dynamic models are all capable of simulating relative permeability curves. However, at high capillary number, only the quasi static and dynamic network model can mimic the effect of low interfacial tension.

\section{ACKNOWLEDGEMENTS}

We gratefully acknowledge financial support from the China National Key Basic Research Development Program under grant (2005cb221304).

\section{REFERENCES}

1. Helge K. D., Michael A. C. A Dynamic Network Model for Two-phase Immiscible Flow. Computational Geosciences, 1998

2. Ji-Youn Arns, Vanessa R. Effect of network topology on relative permeability. Transport in Porous Media, 2004; 55: 33-38

3. Blunt MJ, King P. Relative permeability from two and three dimensional pore scale network modeling. Transport in Porous Media, 1991; 6: 407-433

4. Fatt I. The network model of porous media, I, Capillary pressure characteristics. Pet. Trans. AIME., 1956; 207: 144-159

5. Koplik J, Lasseter TJ. Two-phase Flow in Random Network Models of Porous Media. Society Petroleum Engineering Journal, Feb, 1985; 89-100

6. Morrow NR, Chatzis I, Lim H. Relative Permeabilities at reduced residual saturations. Journal Canadian Petroleum Technology, 1985; 62-69 